\title{
LINFOMA TESTICULAR PRIMARIO. APORTACIÓN DE UN NUEVO CASO Y REVISIÓN DE LA LITERATURA
}

\author{
I. GÓMEZ GARCÍA, R. RODRÍGUEZ PATRÓN, E. SANZ MAYAYO, \\ J.M. RODRÍGUEZ LUNA, A. PALMEIRO URIACH*, S. CONDE SOMESO**, \\ F.J. BURGOS REVILLA, D. GARCÍA ORTELLS, A. ESCUDERO BARRILERO \\ Servicio de Urología. *Servicio de Anatomía Patológica. \\ **Servicio de Cirugía General. Hospital Ramón y Cajal. Madrid.
}

Actas Urol Esp. 28 (2): 141-146, 2004

\section{RESUMEN \\ LINFOMA TESTICULAR PRIMARIO. APORTACIÓN DE UN NUEVO CASO Y REVISIÓN DE LA LITERATURA}

El linfoma testicular primario es un tumor testicular infrecuente, suponiendo no más del 9\% de los tumores testiculares en las series con mayor incidencia; a su vez el linfoma testicular como tumor hematopoyético es infrecuente, con una incidencia del $1 \%$ de los linfomas, pero debido a su histopatología en la mayoría de los casos de alta malignidad, les hace ser de los tumores testiculares más agresivos. La edad de aparición es por encima de los 60 años, convirtiéndose en el tumor más frecuente para este grupo de edad. La falta de series amplias, hace que no exista un protocolo establecido para el tratamiento de esta patología. Presentamos un nuevo caso, realizando revisión de la bibliografía presentando las tendencias terapéuticas actuales para este tipo de patología.

PALABRAS CLAVE: Linfoma testicular primario. Linfoma no Hodgkin. Linfoma extranodal.

\section{ABSTRACT}

PRIMARY TESTICULAR LYMPHOMA. CONTRIBUTION OF ONE CASE AND LITERATURE REVIEW

Primary testicular lymphoma is an uncommon testicular tumour that accounts for no more than $9 \%$ of all testicular tumours in those series with higher incidence; testicular lymphoma as haematopoietic tumours are also rare accounting for just $1 \%$ of all lymphomas; but due to their highly malignant histopathology they may become highly aggressive tumours. Patient age at presentation is over 60 years old which makes it the most frequent tumour for this age group. There is no standard protocol to treat this malignancy due to lack of extensive series. We contribute one case and make a literature review discussing the current therapeutic trends for this disease.

KEY WORDS: Primary testicular lymphoma. Non-Hodgkin lymphoma. Extranodal lymphoma. 
L os tumores testiculares suponen el tumor más frecuente en el varón entre los 20 y 35 años, representando sólo entre el 1 y el $2 \%$ de los tumores del hombre, y el 4\% de las neoformaciones genitales. La estirpe histológica más frecuente es la germinal, variando según las series entre el 85\% y el 90,4\%. Dentro de los tumorales germinales los más frecuentes son los seminomatosos $(60-65 \%)^{11}$, aunque en otras series la frecuencia de los tumores germinales no seminomatosos es superior con respecto a la de los seminomatosos ${ }^{12}$. El linfoma testicular constituye entre el 1 y el 9\% de los tumores testiculares según las series ${ }^{3,4,10,15,16}$, siendo el tumor testicular maligno más frecuente en los varones mayores de 50 años, representando entre el 25 y $50 \%$ de los casos. La afectación testicular secundaria por linfoma no Hodgkin se encuentra entre el $0,14 \%$ y el 1,25\%, que aumenta hasta el $4 \%$ en linfomas tipo Burkitt ${ }^{7}$, encontrándose infiltración linfoide en el 18\% de las necropsias.

\section{CASO CLÍNICO}

Varón de 71 años de edad que presenta dolor testicular derecho, no irradiado, de tres meses de evolución, con aumento del diámetro y de la consistencia testicular en el último mes. Presenta síndrome constitucional, con pérdida de $3 \mathrm{~kg}$ de peso en el último mes. Fiebre en picos de hasta $39^{\circ} \mathrm{C}$, sudación profusa, no-clínica miccional, nohematuria. Como antecedentes de interés destaca EPOC con tratamiento con inhaladores y oxígeno crónico domiciliario.

En la exploración física, el paciente está afectado, con palidez mucocutánea, crepitantes en la auscultación pulmonar, edemas en miembros inferiores, no adenopatías, hematomas en ambos miembros superiores. La exploración genital en el hemiescroto derecho presenta edema de cubiertas, aumento de la consistencia del epidídimo y del teste, con desaparición del surco epidídimo testicular, sin signos de fluctuación ni de abcesificación. Tacto rectal próstata adenomatosa grado II-III/IV.

Analíticamente destaca hemoglobina de 8,1 gr/dl, (previa de 13,5 gr/dl), plaquetas 51000, 9100 leucocitos. Coagulación elevación del fibrinógeno 1500 (límites de la normalidad inferior a 400). Lactato deshidrogenosa (LDH) elevada, B-
HCG, CEA, AFP, función renal, hepática y pancreática normales. Radiografía de tórax, mínima congestión vascular, radiografía de abdomen normal.

Ecografía renal y vesical sin alteraciones, próstata de 4,5 × 4 × 4,1 cm. Ecografía testicular, testículo izquierdo normal, testículo derecho se distingue hasta tres áreas heterogéneas con disminución de la ecogenicidad, sin calcificaciones (Figs. 1 y 2) que afectan a polo superior, inferior y epidídimo, no-hidrocele.

Con la sospecha diagnóstica de absceso testicular versus tuberculosis testicular, brucelosis o tumor testicular, realizamos orquiectomía radical por vía inguinal.

La anatomía patológica informa, en la macroscópica de tumor testículo de $8 \times 6 \times 4 \mathrm{~cm}$, presentando al corte masa blanquecina lobulada de

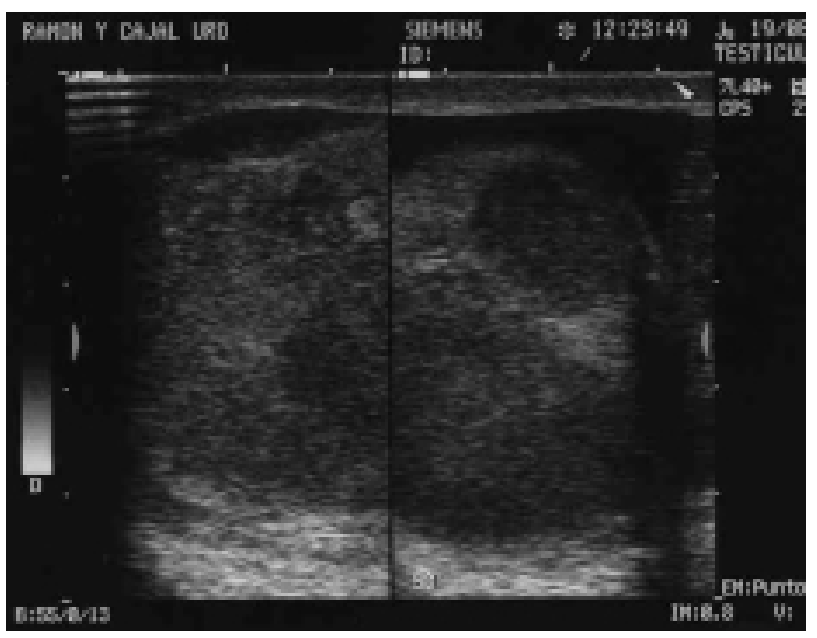

FIGURA 1. Ecografia testicular: afectación testicular difusa.

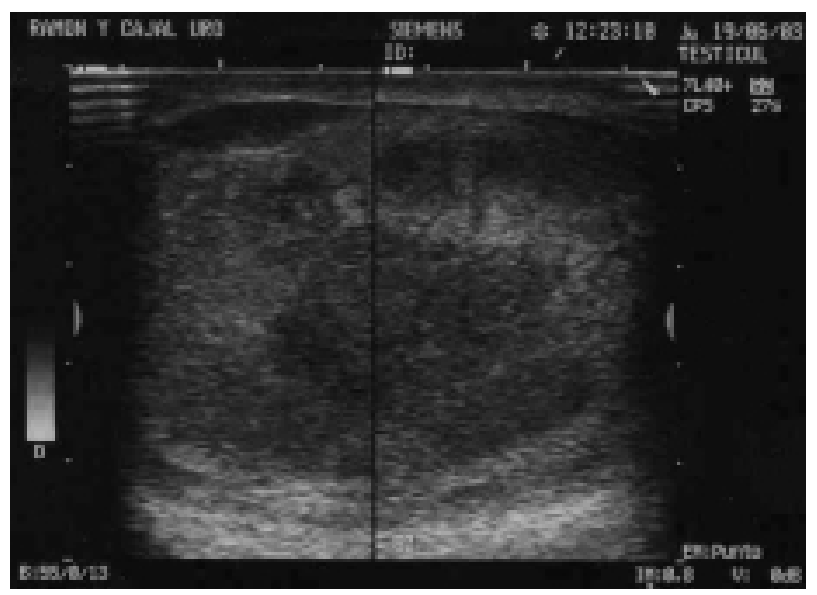

FIGURA 2. Ecografia testicular: áreas hipoecogénicas. 
$5,5 \mathrm{~cm}$, que infiltra albuginea y epidídimo pero que no sobrepasa la capa externa ni el borde hiliar (Fig. 3). Microscópicamente el testículo está afectado por linfoma difuso B de células grandes (Fig. 4), con alto índice de proliferación (minb1 > 80\%), y marcadores CD 20+ (Fig. 5). Estadio 1E-B.

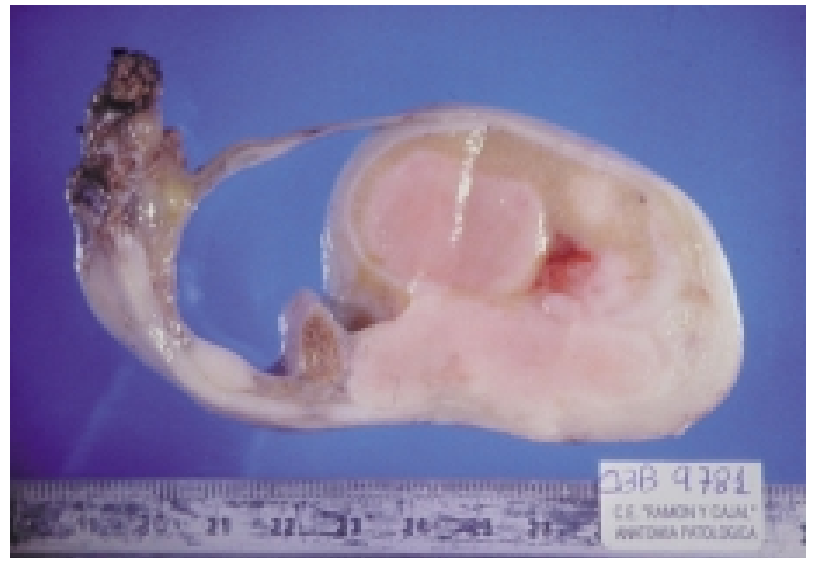

FIGURA 3. Pieza de orquiectomía.

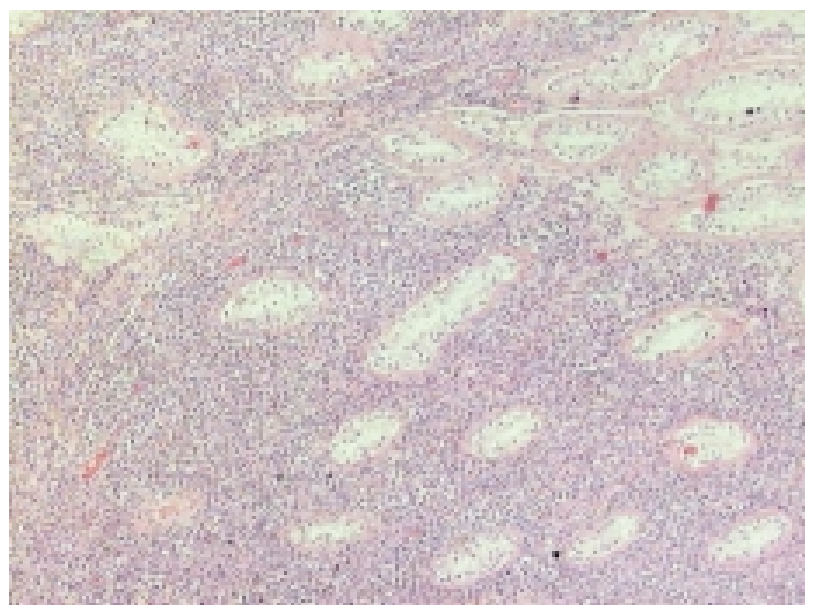

FIGURA 4. Microscopia: tumor de células de mediano tamaño que infiltra estroma sin afectación de túbulos seminiferos.

Con el diagnóstico de linfoma testicular primario, solicitamos TAC de cuerpo completo de estadiaje, apreciándose esplenomegalia homogénea, adenopatías retroperitoneales aisladas inferiores a $1 \mathrm{~cm}$, como hallazgos destacables.

En la evolución post-quirúrgica el paciente presenta sudación profusa, fiebre en picos, astenia, y edemas de los miembros inferiores. Analíticamente persiste, anemia $8,9 \mathrm{gr} / \mathrm{dl} \mathrm{de}$ hemoglobina, plaquetas de 37000 , y fórmula leucocitaria y linfocitaria normales.

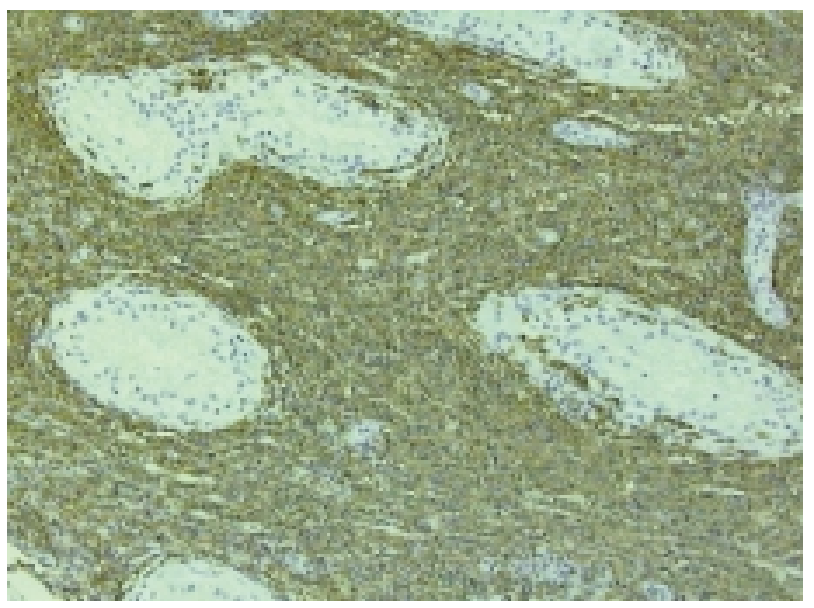

FIGURA 5. Tinción positiva para células de la serie B (CD 20+).

Con el diagnóstico de síntomas $\mathrm{B}$ en paciente con linfoma testicular primario, realizamos biopsia de medula ósea, obteniéndose un cilindro de $3 \times 0,3 \mathrm{~cm}$, en la que existe médula ósea hipercelular con cambios regenerativos y displásicos, a expensas de la serie granulocítica. Se evidencia marcada plasmocitosis intersticial, infiltración por células linfoblásticas (Fig. 6).

Al no evidenciarse infiltración de la médula ósea, realizamos frotis sanguíneo para justificar la causa de la anemia y trombocitopenia. Se aprecia agregación plaquetaria, realizándose test de Coombs directo siendo débilmente positivo a inmunoglobulina G, siendo la causa de la agregación.

Durante su ingreso hospitalario el paciente empieza con disfonía y disfagia realizándose fibros-

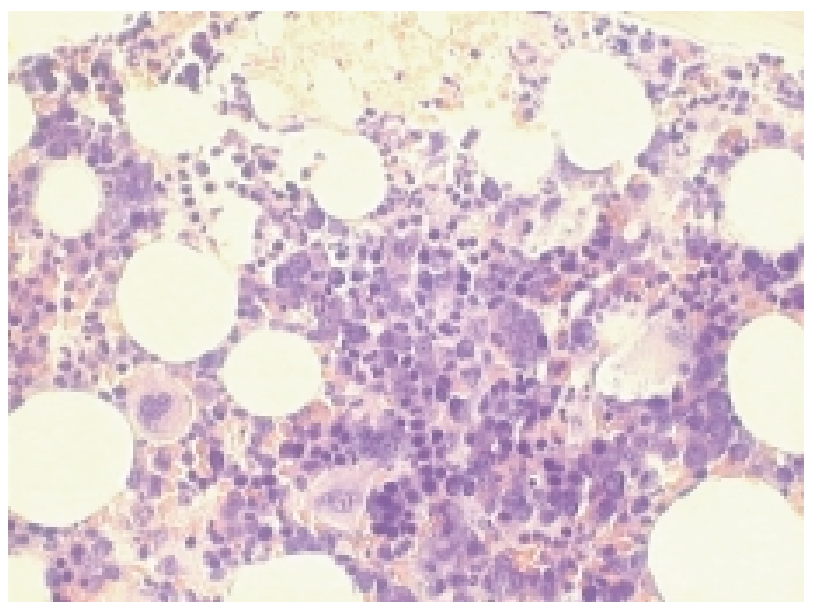

FIGURA 6. Médula ósea: infiltración por células de estirpe linfoblástica. 
copia, observándose lesión polipoidea en seno piriforme. El TAC cervical normal.

Durante el ingreso al paciente se le pauta Rituximab y al día siguiente el primer ciclo de CHOP (ciclofosfamida, doxorrubicina, vincristina, prednisona), presentando intensa mejoría de los síntomas B, con desaparición de la fiebre. Se instaura tratamiento con quimioterapia pautándosele 6 ciclos de R-CHOP. Se le administró radioterapia genital coadyuvante y quimioprofilaxis cerebral. A los 12 meses el paciente se encuentra libre de enfermedad.

\section{DISCUSIÓN}

En 1877 Malassez describe por primera vez el linfoma testicular primario no Hodgkin ${ }^{1}$. Los linfomas testiculares primarios representan aproximadamente el $1 \%$ de los linfomas no Hodgkin ${ }^{2}$, sin embargo es más usual la afectación secundaria testicular por linfoma pudiendo aparecer en más del 4\% de los linfomas tipo Burkitt. El linfoma testicular constituye entre el 1 y el $9 \%$ de los tumores testiculares según las series $3,4,15,16$, siendo el tumor testicular maligno más frecuente en los varones mayores de 60 años, representando entre el 25 y $50 \%$ de los tumores testiculares en ese grupo de edad; el linfoma testicular en la infancia es excepcional habiéndose descrito hasta el momento sólo 9 pacientes $^{14}$. Se han informado caso de linfoma testicular primario bilateral, ocurriendo hasta en el $23 \%$ de los pacientes con linfoma testicular ${ }^{4}$.

La etiología del linfoma testicular primario es desconocida, sin conocerse factores predisponentes, aunque se ha descrito probable relación con orquitis crónica, criptorquidia $\mathrm{y}$ filariasis ${ }^{16}$. $\mathrm{Al}$ igual que otros linfomas extragonadales, se está produciendo un aumento de la incidencia en pacientes con el síndrome de inmunodeficiencia adquirida (SIDA); siendo el $25 \%$ de los tumores testiculares en estos pacientes. En enfermos de SIDA, el linfoma testicular primario tiene una edad de aparición más joven, una histología más agresiva, y un peor pronóstico ${ }^{17}$.

La presentación clínica más habitual, dolor testicular a la palpación y espontáneo, con aumento del volumen y de la consistencia testicular. Con diagnóstico previo habitual de orquioepididimitis crónica $^{3,5,10,15,16}$, presentándose en ocasiones excepcionales como masa testicular indolora ${ }^{7}$. El síndrome constitucional está presente entre un 25 y $41 \%$ de los pacientes ${ }^{16}$. En la exploración es habitual encontrar un testículo agrandado, con aumento de la consistencia, ocasional hidrocele, con un epidídimo nodular y pétreo. Analíticamente la LDH está elevada, correlacionándose directamente con la actividad tumoral. Otros marcadores tumorales como la AFP, B-HCG, CEA son normales.

La clasificación patológica de los linfomas no Hodgkin, es fundamental el subtipo histológico y menos importante el estadiaje, ya que del primero se basan las opciones terapéuticas. Rappaport hizo la primera clasificación basándose en la arquitectura ganglionar (nodular o difuso) y en la microscopía a gran aumento (bien diferenciado, escasamente diferenciado, histiocítico). El linfoma nodular presenta características de los ganglios linfáticos normales, formando centros germinales, mientras en los difusos la arquitectura ganglionar normal no existe. Posteriormente al esquema de Rappaport se aunaron todas las clasificaciones en una, la Working Formulation, dividiendo los linfomas no Hodgkin en grado bajo, intermedio y alto. Así los linfomas de bajo grado presentan una buena evolución mientras que los de grado intermedio y alto sin tratamiento presentan supervivencia muy corta. Histológicamente, el linfoma testicular se caracteriza, por un aumento difuso del teste, con infiltración del cordón y del epidídimo, pero sin afectación de la túnica vaginal ${ }^{18}$. La estirpe celular linfoide suele ser de tipo B aunque se han descrito de manera excepcional estirpe tipo $\mathrm{T}^{12,13,15}$. La totalidad de los linfomas testiculares, son de tipo difuso, siendo hasta el $90 \%$ de ellos de grado medio-alto de malignidad $^{6}$. Dado que este tipo de linfomas son de origen extranodal, con afectación difusa y en el $90 \%$ de los casos con grado medio-alto de malignidad, le confiere un peor pronóstico que otro tipo de linfomas, y una mayor tendencia a la afectación del SNC.

La diferenciación con los tumores testiculares de origen embrionario se debe realizar mediante la utilización de marcadores monoclonales de estirpe epitelial.

Ecográficamente, se puede observar afectación focal o difusa de áreas con disminución de la eco- 
genicidad, en testículos aumentados de tamaño ${ }^{5}$. De manera puntual se han descrito casos de linfomas que ecográficamente, presentan un aumento de la ecogenicidad. El diagnóstico diferencial ecográfico debe realizarse con seminomas, orquitis crónica piógena, brucelosis testicular, tuberculosis testicular, rabdomiosarcoma y leucemia.

Los factores pronósticos más importantes son la edad y el estadio tumoral (Tabla I), siendo la estirpe histológica un factor pronóstico secundario $^{6}$, ya que la mayor parte de los casos son de alto grado de malignidad ${ }^{8}$. Los factores con un mal pronóstico son, edad avanzada, presencia de síntomas $\mathrm{B}$, tumor primario mayor de $9 \mathrm{~cm}$, grado de esclerosis, infiltración de epidídimo, cordón o vasos. Los factores más importantes en el pronóstico son, el estadio tumoral (tumores con estadio IE o IIE, presentan mejor pronóstico que los estadios III y IV), el grado histológico, y el tiempo libre de enfermedad. Los linfomas testiculares primarios tienen una evolución agresiva con una supervivencia a los 5 años entre el 10 y el $40 \%$ según las series ${ }^{3,6,7,10,16,18}$. Tienen tendencia a la diseminación rápida, con un $50 \%$ de recidiva en los dos primeros años, afectando áreas extraganglionares como anillo de Waldeyer, sistema nervioso central, piel, pulmón y médula ósea, ensombreciendo el pronóstico $3,6,7,10,16$.

Para el tratamiento del linfoma testicular primario, disponemos de la cirugía, radioterapia, quimioterapia y profilaxis quimioterapéutica del sistema nervioso central. Dada la rareza de esta enfermedad no existe un protocolo estandarizado

\section{TABLA I}

ESTADIAJE ANN ARBOR DE LINFOMAS NO HODGKING

\begin{tabular}{|l|l|}
\hline Estadio IE & $\begin{array}{l}\text { Afectación de una única localización } \\
\text { extralinfática. }\end{array}$ \\
\hline Estadio IIE & $\begin{array}{l}\text { Afectación de más de una localización } \\
\text { extralinfática a un lado del diafragma. }\end{array}$ \\
\hline Estadio IIE & $\begin{array}{l}\text { Afectación de más de una región } \\
\text { extralinfática a ambos lados del } \\
\text { diafragma. }\end{array}$ \\
\hline Estadio IVE & $\begin{array}{l}\text { Afectación de diseminada de órganos } \\
\text { extralinfáticos con o sin afectación } \\
\text { ganglionar. }\end{array}$ \\
\hline Se añade “B”, cuando están presentes los síntomas B \\
\hline
\end{tabular}

para su tratamiento.

La orquiectomía radical está indicada tanto en estadios localizados como en estadios avanza$\operatorname{dos}^{2,4,10,14-16,18}$. La orquiectomía es el primer escalón terapéutico en estadios localizados (IE, IIE), mientras que en estadios avanzados se puede post-poner al tratamiento sistémico ${ }^{16}$. El papel de la orquiectomía radical bilateral profiláctica es controvertido, estando descartada, siempre y cuando se asocie tratamiento quimioterapéutico coadyuvante ${ }^{7,18}$. La orquiectomía en enfermedad localizada como único tratamiento, conlleva a riesgo de recidiva en menos de dos años en más del 90\% de los casos $2,5,6,9,14,19$.

El tratamiento con radioterápico (25003500cGY) sobre el área genital es controvertido, existiendo autores en los que sus resultados son los mismos con o sin radioterapia coadyuvan$t^{7,9,18}$, dejando estos autores sólo la radioterapia para los estadios IA y cuando existe gran volumen tumoral; series más amplias ${ }^{10}$ han conseguido excelentes resultados asociando ciclos cortos de quimioterapia (3 ciclos de $\mathrm{CHOP}$ ) con radioterapia de la zona afecta. La justificación del uso de la radioterapia evita laparotomía de estadiaje, previene la aparición de linfoma contralateral, y la toxicidad quimioterápica es menor en pacientes jóvenes ${ }^{10}$. La radioterapia sola no es efectiva, debiendo ser aplicada como único tratamiento en pacientes que no son candidatos a quimioterapia sistémica ${ }^{16}$.

La quimioterapia (QT) y la cirugía debe ser la base del tratamiento. La QT en estadios localizados es un tema controvertido, pero en la mayoría de las series se ha demostrado que el tratamiento con quimioterapia aumenta el porcentaje de remisiones completas ${ }^{10,15,16,19}$. La pauta actual, consiste en 6 ciclos de poliquimioterapia con RCHOP o B-CHOP ${ }^{8-11,14,16,19 . ~}$

En el tratamiento en estadios avanzados III y IV, el tratamiento es la quimioterapia (CHOP), pero aún así la esperanza de vida no suele ser superior a 2 años, existiendo casos aislados de remisión completa ${ }^{15,16,19}$.

La quimioprofilaxis del sistema central está indicada en estadios avanzados (III y IV), afectación de la médula ósea, y en pacientes jóvenes ${ }^{10,14,15}$.

La recidiva testicular, en el testículo contralateral es entre el 8 y 35\%, existiendo centros en 
que se aplica radioterapia escrotal tras la orquiectomía, reduciendo el número de recurren$\operatorname{cias}^{16,19}$.

\section{CONCLUSIONES}

El linfoma testicular primario es un tumor infrecuente de testículo no suponiendo más del 9\% en las series más amplias. La localización extranodal le confiere un mal pronóstico, existiendo recidiva en estadios localizados en más del 90\% de los casos antes de dos años. El diagnóstico suele ser tardío y demorarse por confundir en la mayoría de los casos con la orquitis bacteriana crónica. El tratamiento de elección en los estadios localizados es la orquiectomía, con o sin radioterapia, y quimioterapia; en los estadios avanzados el tratamiento fundamental es la quimioterapia con orquiectomía. La quimioprofilaxis del sistema nervioso central está indicada en pacientes jóvenes, infiltración de médula ósea, y en estadios avanzados. El papel de la radioterapia es controvertido, estando la mayoría de las series de acuerdo en el tratamiento coadyuvante en estadios IE.

\section{REFERENCIAS}

1. TEPPERMAN BS, GOSPODAROWICZ MK, BUSH RS.: Non-Hodgkin lymphoma of the testis. Radiology 1982 jan; 142 (1): 203-208.

2. CHARNSANGAVEJ C.: Lymphoma of the genitourinary tract. Radiol Clin North Am 1990 jul; 28 (4): 865-877.

3. DUNCAN PR, CHECA F, GOWING NF.: Extranodal non-Hodgkin's lymphoma presenting in the testicle: a clinical and pathologic study of 24 cases. Cancer 1980 apr 1; 45 (7): 1578-1584.

4. TURNER RR, COLBY TV, MACKINTOSH FR.: Testicular lymphomas: a clinicopathologic study of 35 cases. Cancer 1981 nov 1; 48 (9): 2095-2102.

5. MOORJANI V, MASHANKAR A, GOEL S.: Sonographic appearance of primary testicular lymphoma. Am J Roentgenol 1991 dec; 157 (6): 1225-1226.

6. SUTCLIFFE SB.: Clinical features and management of localized extranodal lymphomas. Haematological oncology. Cambridge: Cambridge University Press 1992: 189-222.
7. ASENSIO AJ, BESSES C, PALACIN A.: Linfoma primario testicular. Presentación de 3 casos. Med Clin 1994 sep 24; 103 (9): 339-341.

8. MARTENSON JA, BUSKIRK SJ, ILSTRUP DM. Patterns of failure in primary testicular nonHodgkin's lymphoma. J Clin Oncol 1988 feb; 6 (2): 297-302.

9. MONTSERRAT E.: Tratamiento de los linfomas nohodgkianos. Med Clin (Barc) $1990 \mathrm{dec}$ 1; 95 (19): 743-752.

10. CONNORS JM, KLINO P, VOSS N.: Testicular lymphoma: improved outcome with early brief chemotherapy. J Clin Oncol 1988 may; 6 (5): 776-781.

11. RICHIE JP.: Neoplasias testiculares. En: Campbell de Urología. Ed. Panamericana 1994; 30: 12201262. Argentina.

12. SOTO DELGADO M, VARO SOLÍS C, JUAREZ SOTO A.: Consideraciones sobre los tumores de testículo: nuestra experiencia. Arch Esp Urol 1999 mar; 52 (2): 123-131; discussion 132.

13. VARO SOLÍS C, BACHILLER BURGOS J, SOTO DELGADO M.: Linfoma primario de testículo. Actas Urol Esp 1997 sep; 21 (8): 781-784.

14. PAKZAD K, MACLENNAN T, ELDER S.: Follicular large celll lymphoma localized to the testis in children. J Urol 2002 jul; 168 (1): 225-228.

15. RODRÍGUEZ MORENO MV, MARTINO ML.: Linfoma testicular primario. Presentación de dos casos. Rev Clin Esp 1995 nov; 195 (11): 769-772.

16. SHAHAB N, DOLL DC.: Testicular lymphoma. Semin Oncol 1999 jun; 26 (3): 259-269.

17. LEIBOVITCH I, GOLDWASSER B.: The spectrum of acquired immune deficiency syndrome-associated testicular disorders. Urology 1994 dec; 44 (6): 818824.

18. FERRY JA, HARRIS ML, YOUNG RH.: Malignant lymphoma of the testis, epididymis, and spermatic cord. A clinicopathologic study of 69 cases with immunophenotypic analysis. Am J Surg Pathol 1994 apr; 18 (4): 376-390.

19. CRELLIN AM, HUDSON BV, BENNETT MH.: NonHodgkin's lymphoma of the testis. Radiother Oncol 1993 may; 27 (2): 99-106.

Dr. I. Gómez García

C/ María Tubau, 15 - portal 3 - 1 3

28050 Madrid

(Trabajo recibido el 30 septiembre de 2003) 